\title{
Research on Speed Control of Brushless DC Motor Based On Predictive PI Control
}

\author{
Yan Li-mei, Tang Qi-hao \\ (Department of Electrical Engineering and Information, Northeast Petroleum University, Heilongjiang Daqing, \\ 163318, China)
}

\begin{abstract}
The traditional PI control method is used to control the brushless DC motor with the disadvantage that the proportional and integral parameters are constant. So an advanced method of predictive PI control is proposed, which predicts the value of speed of next moment by multi-order incremental method based on the current and previous speed of brushless DC motor. And the simulation model is established by MATLAB. Then the results that the method is better than traditional PI control and fuzzy control in the speed modulation, specific performances: faster response, less overshoot, stable anti-disturbance and better stability.
\end{abstract}

Keywords: Brushless DC motor; Multi-order incremental method; Predictive PI control

\section{Introductions}

In today's era, the use of electric energy has been very common, the electric motor as the electric energy to mechanical energy conversion device accompanied by the widespread use of electrical energy also has been a wide range of popularity and rapid development ${ }^{[1]}$.In industrial production, compared with the Ac motor, the brushless DC motor has the advantages of low noise, long service life, high efficiency, compact structure, easy to control and so on ${ }^{[2-3]}$.The speed regulating systems built by brushless DC motor are often nonlinear and strong coupling control systems, the classical PID control has many advantages, such as simple principle, small steady state error and so on ${ }^{[4]}$.But there are some limitations and deficiencies, which is that it has better control effect for some linear time invariant systems, and for the control of nonlinear systems, PID control is not good enough ${ }^{[5]}$. No matter how parameters are set, the control effect is not ideal ${ }^{[6]}$, so it is very necessary to study a new control method of Brushless DC motor.

\section{Mathematical Model Of Brushless DC Motor}

The voltage balance equation of three-phase winding of Brushless DC motor is as follows ${ }^{[7]}$

$\left[\begin{array}{l}u_{A} \\ u_{B} \\ u_{C}\end{array}\right]=\left[\begin{array}{ccc}R_{S} & 0 & 0 \\ 0 & R_{S} & 0 \\ 0 & 0 & R_{S}\end{array}\right]\left[\begin{array}{l}i_{A} \\ i_{B} \\ i_{C}\end{array}\right]+$
$\left[\begin{array}{ccc}L_{A} & L_{A B} & L_{A C} \\ L_{B A} & L_{B} & L_{B C} \\ L_{C A} & L_{C B} & L_{C}\end{array}\right] \frac{\mathrm{d}}{\mathrm{d} t}\left[\begin{array}{l}i_{A} \\ i_{B} \\ i_{C}\end{array}\right]+\left[\begin{array}{l}e_{A} \\ e_{B} \\ e_{C}\end{array}\right]$

$u_{\mathrm{A}}, u_{\mathrm{B}}, u_{\mathrm{C}}$ are stator winding voltage $(\mathrm{V}) ; i_{\mathrm{A}}, i_{\mathrm{B}}, i_{\mathrm{C}}$ are stator phase winding current $(\mathrm{A}) ; e_{\mathrm{A}}, e_{\mathrm{B}}, e_{\mathrm{C}}$ are stator phase winding electromotive force $(\mathrm{V})$; It is assumed that the resistance of the three-phase winding is equal to $R_{\mathrm{S}} . L_{\mathrm{A}}=L_{\mathrm{B}}=L_{\mathrm{C}}$ are three-phase winding self-inductance $(\mathrm{H}) ; L_{\mathrm{AB}}$ is the mutual inductance between the A phase and the B phase $(\mathrm{H})$, and $L_{\mathrm{AB}}=L_{\mathrm{BA}}, L_{\mathrm{AC}}=L_{\mathrm{CA}}, L_{\mathrm{BC}}=L_{\mathrm{CB}}$ 。

The salient pole effect can be neglected for the convex loaded rotor structure ${ }^{[8]}$. That is

$L_{A}=L_{B}=L_{C}=L$

$L_{A B}=L_{B C}=L_{C A}=L_{C B}$

$=L_{A C}=L_{C B}=L_{B A}=M$

Formula (1) can be rewritten into

$\left[\begin{array}{l}u_{A} \\ u_{B} \\ u_{C}\end{array}\right]=\left[\begin{array}{ccc}R_{S} & 0 & 0 \\ 0 & R_{S} & 0 \\ 0 & 0 & R_{S}\end{array}\right]\left[\begin{array}{l}i_{A} \\ i_{B} \\ i_{C}\end{array}\right]+$
$\left[\begin{array}{ccc}L & M & M \\ M & L & M \\ M & M & L\end{array}\right] \frac{\mathrm{d} p}{\mathrm{~d} p}\left[\begin{array}{c}i_{A} \\ i_{B} \\ i_{C}\end{array}\right]+\left[\begin{array}{c}e_{A} \\ e_{B} \\ e_{C}\end{array}\right]$ 
When the three-phase winding of the stator is connected with the Y,

$i_{A}+i_{B}+i_{C}=0$

We can get

$M i_{B}+M i_{C}=-M i_{A}$

Put the formula (5), (6) into formula (4), we can get

$\left[\begin{array}{l}u_{A} \\ u_{B} \\ u_{C}\end{array}\right]=\left[\begin{array}{ccc}R_{S} & 0 & 0 \\ 0 & R_{S} & 0 \\ 0 & 0 & R_{S}\end{array}\right]\left[\begin{array}{l}i_{A} \\ i_{B} \\ i_{C}\end{array}\right]+$

$\left[\begin{array}{ccc}L-M & 0 & 0 \\ 0 & L-M & 0 \\ 0 & 0 & L-M\end{array}\right] \frac{\mathrm{d}}{\mathrm{d} p}\left[\begin{array}{c}i_{A} \\ i_{B} \\ i_{C}\end{array}\right]+\left[\begin{array}{c}e_{A} \\ e_{B} \\ e_{C}\end{array}\right]$

\section{Predictive PI Control Method}

\section{1 predictive PI control theory}

Predictive PI control is the PI control which guides the current moment using the prediction speed of Brushless DC motor at the next time. The predicted speed of the motor is obtained, do the subtraction with the value of the given speed and the value of the predicted motor speed, and the corresponding error is obtained ${ }^{[9]}$. This error is obtained from the predicted speed of the motor and the given speed value, so it is predicted error value. The predicted error value is adjusted by the PI control, and then the entirely improve the control effect of the brushless DC motor, the principle diagram of the predictive PI control is shown in figure 1.

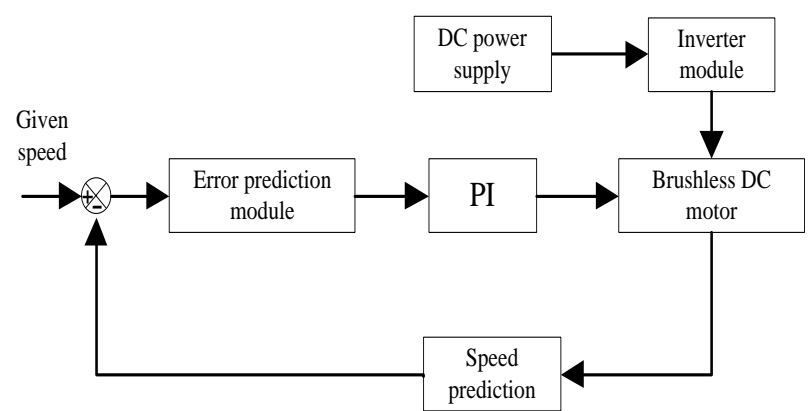

Fig. 1 Schematic diagram of predictive PI control

\subsection{Multi order increment prediction}

The key of the predictive PI control method is to obtain the speed value of the brushless DC motor at the next moment. This paper adopts the multi - order incremental method to predict the rotational speed. The multi order incremental method is derived from the first order and the two order.

\section{(1) First order incremental method}

The first order increment prediction method is the simplest and most basic prediction method in the multi-order incremental forecasting method, and the principle diagram of the first - order incremental prediction method is shown in figure 2 .

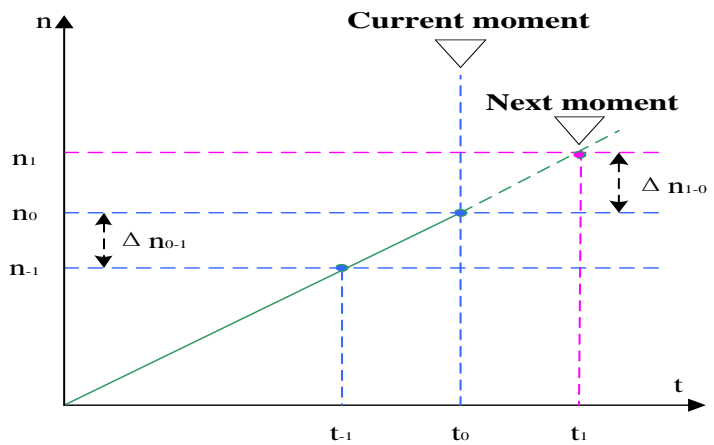

Fig. 2 Schematic diagram of first order incremental method

In figure 2, the horizontal axis $t$ represents time, the vertical axis $n$ represents the rotational speed of the motor, $t_{0}$ represents the current time, the corresponding longitudinal coordinate $n_{0}$ represents the true speed of 
the motor at present, $t_{-1}$ represents the last moment, the corresponding $n_{-1}$ represents the true speed of the motor at the last moment, $t_{1}$ represents the next moment, the corresponding $n_{1}$ represents the true speed of the motor at the next moment, that is the value of the motor speed to be predicted, Their time intervals are equal and $T=t_{1}-t_{0}=t_{0}-t_{-1}$, Then the speed increment between $t_{0}$ moment and $t_{-1}$ moment is:

$\Delta n_{0-1}=n_{0}-n_{-1}$

The speed increment between the $t_{1}$ moment and the $t_{0}$ moment is :

$\Delta n_{1-0}=n_{1}-n_{0}$

Use the first order increment to predict, it can be considered:

$\Delta n_{1-0}=\Delta n_{0-1}$

According to (10) the motor speed of the $t_{1}$ moment can be obtained in the first order:

$n_{1}=n_{0}+\Delta n_{1-0}=2 n_{0}-n_{-1}$

\section{(2) The $n$ order promotion of the multi-order incremental method}

In the process of $n$ order rotational speed prediction, it is mainly divided into three parts, which are the ascending order calculation, the order increment prediction, and the reduced order prediction. According to the order number determine the required actual speed value of the motor at the current moment and before the moment, $t_{0}$ represents the current moment, the corresponding longitudinal coordinate $n_{0}$ represents the true speed of the motor at present, $t_{-1}$ represents the last moment. The corresponding $n_{-1}$ represents the true speed of the motor at the last moment, $t_{-2}$ represents the last two moments, the corresponding $n_{-2}$ represents the true speed of the motor at the last two moments, until $t_{\text {-n }}$ moment, the speed corresponds to this moment $n_{\mathrm{n}}, t_{1}$ represents the next moment, the corresponding $n_{1}$ represents the true speed of the motor at the next moment, and the selected moments meet the conditions:

$T=t_{1}-t_{0}=t_{0}-t_{-1}=\ldots=t_{-(n-1)}-t_{-n}$

The corresponding first order increment is calculated according to the known speed value:

$\left\{\begin{aligned} \Delta n_{0-1} & =n_{0}-n_{-1} \\ \Delta n_{-1-2} & =n_{-1}-n_{-2} \\ & \cdots \cdots \\ \Delta n_{-(n-1)-n} & =n_{-(n-1)}-n_{-n}\end{aligned}\right.$

The total number of the known motor speed value is $\mathrm{N}$, the total number of the calculated first order increment is $n-1$. Based on the first order incremental value of the calculated rotational speed, the two order increment is calculated:

$\left\{\begin{array}{c}\Delta^{2} n_{0-2}=\Delta n_{0-1}-\Delta n_{-1-2} \\ \Delta^{2} n_{-1-3}=\Delta n_{-1-2}-\Delta n_{-2-3} \\ \cdots \cdots \\ \Delta^{2} n_{-(n-2)-n}=\Delta n_{-(n-2)-(n-1)}-\Delta n_{-(n-1)-n}\end{array}\right.$

The calculated two order incremental value of motor speed is a total of $n-2$. Based on the calculation of the two order incremental value, the same law is used to calculate the rotational speed of the three order until the $\mathrm{n}$ order increment, the calculation of the number of $n$ order increment is one, and its value is:

$\Delta^{n} n_{0-n}=\Delta^{n-1} n_{0-(n-1)}-\Delta^{n-1} n_{-1-n}$

All the $n$ order incremental calculation is completed, for the $\mathrm{n}$ order speed prediction, it can be considered:

$\Delta^{n} n_{1-(n-1)}=\Delta^{n} n_{0-1}$

Do reduced order calculation and calculate the predicted value of the rotational speed, first do $n-1$ order of reduced order calculation:

$\Delta^{n-1} n_{1-(n-2)}=\Delta^{n} n_{1-(n-1)}+\Delta^{n-1} n_{0-(n-1)}$

Do the reduced order calculation until the first order increment of the rotational speed is obtained.:

$\Delta n_{1-0}=\Delta^{2} n_{1-1}+\Delta n_{0-1}$

The predicted value of the motor speed of the next moment can be obtained according to the true value of the rotational speed and rotational speed first order increment of reduced order.

$n_{1}=\Delta n_{1-1}+n_{0}$ 
In theory, with the increase of the order number, the fitting degree of the predicted motor speed to the real speed of the motor is higher. It can be seen that the first order rotational speed prediction method is suitable for the linear variation of the speed curve, and the high order incremental prediction is more suitable for the curve change.

\section{Simulation Verification}

The rotational speed prediction model established by MATLAB is shown in Figure 3. Brushless DC motor stator winding phase resistance $R=0.35 \Omega$, the stator winding inductance and mutual inductance respectively are $L=0.0036 \mathrm{H}, M=0.00023 \mathrm{H}$, the moment of inertia is set to $J=0.0022 \mathrm{~kg} \cdot \mathrm{m}^{2}$, The rated speed of the motor is $n_{s}=3150 \mathrm{r} / \mathrm{min}$ 。

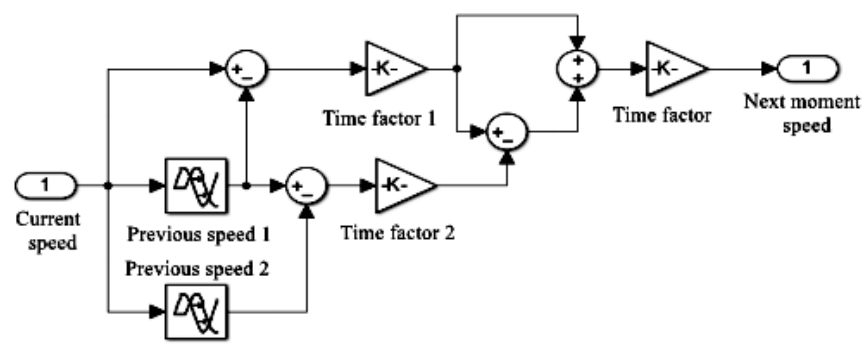

Fig. 3 structure model of rotational speed prediction model

Fig. 4 to Fig.6 are respectively the simulation results of the motor speed in the traditional PID control, fuzzy control and predictive PI control.

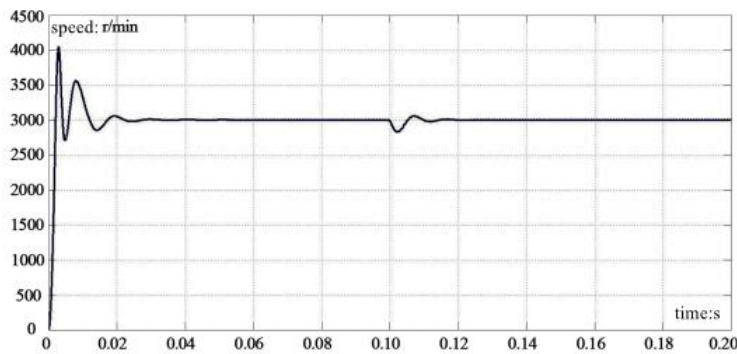

Fig. 4 waveform of motor speed in the traditional PID control

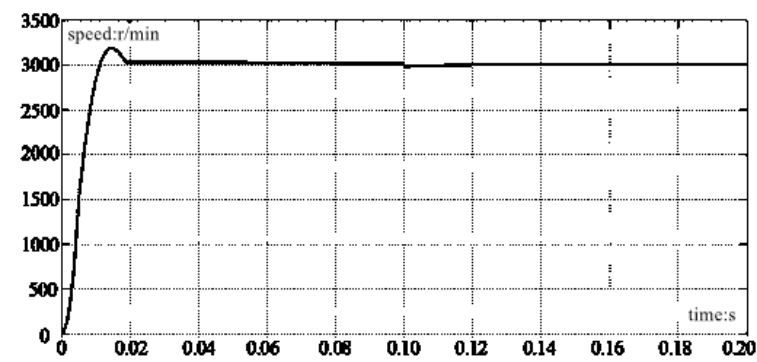

Fig. 5 waveform of motor speed in fuzzy control

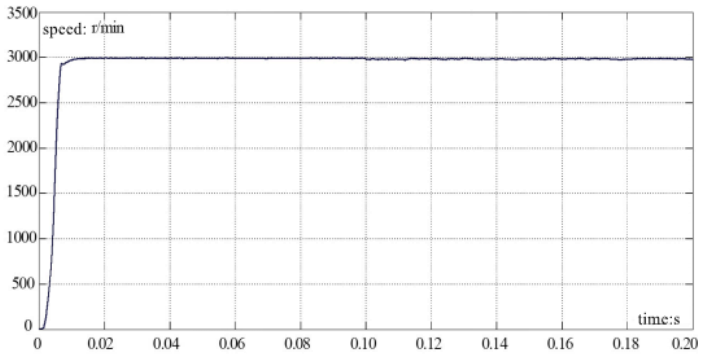

Fig. 6 the waveform of the motor speed in the prediction of PI control 
Through the comparative analysis, from the adjustment time and overshoot of the speed, the effect of the predictive PI control is superior to the classic PID control and fuzzy control. In the ability to resist disturbance, after adding the disturbance signal, the fluctuation of the speed waveform is very small, and quickly return to a given speed value, and the static error of the speed is very small. Predictive PI control can make the brushless DC motor speed better in the rapidity, stability, complexity and other aspects of the effect.

\section{Conclusions}

For some disadvantages of the control method of Brushless DC motor, this paper presents an improved predictive PI control, the rotational speed is predicted by the method of multi-order increment. And the system is modeled and simulated by MATLAB. By comparing the predictive PI control with the classical PID control and fuzzy control, it is proved that the improved predictive PI control has better control effect in the aspects of fast and stable control in Brushless DC motor.

\section{References}

[1]. Xia Changliang, Fang Hongwei. "Permanent-Magnet Brushless DC Motor and Its Control", Transactions Of China Electrotechnical Society, 2012, vol.27, no.3, pp.25-34.

[2]. ZHANG Kui, LEI Yong. "Modeling and Simulation of Brushless DC Motor Intelligent PID Control System", Measurement \& Control Technology, 2015, vol.34, no.4, pp.81-84.

[3]. SHENG Tiantian, WANG Xiaolin, GU Cong, et al. "An Average Torque Control Method for Brushless DC Motors Using Overlap Commutation Strategy", Proceedings of the CSEE, 2015, vol.35, no.15, pp. 3939-3947.

[4]. GUO Wei, ZHANG Ai-hua, CHEN Chen, et al. "Brushless DC Motor Speed Control System Simulation and Control", Computer Simulation, 2015, vol.32, no.10, pp. 360-364.

[5]. Zuo Yuefei, Li Minghui, Zhang Jie, et al. "Influence of Control Gain on Active Disturbance Rejection Controller for PMSM", Transactions of China Electrotechnical Society, 2006, vol.31, no.3,pp. 58-65.

[6]. JIANG Weidong, HU Yang, HUANG Hui, et al. "The Commutation Process Analysis and the Method Reducing Commutation Torque Ripple of Brushless DC Motors Based on the Coordinate Transformation", Proceedings of the CSEE, 2015, vol.35, no.24, pp. 6527-6534.

[7]. Li Hong, Wang Chong-wu. "Design of Fuzzy Adaptive Controller for PMBLDCM", Computer Simulation, 2011, vol.28, no.2, pp. 209-212.

[8]. Wang Xiaoyuan, Fu Tao. "Brushless DC Motor Control System Based on Fuzzy R BF Neural Network", Micromotors, 2015, vol.48, no. 11, pp. 33-36

[9]. Guo Wei, Yu Wenwen, Xia Youliang. "Simulation of Speed Control Strategy for Brushless DC Motor", Computer Simulation, 2015, vol.32, no.9, pp. 372-376 